\title{
Time-Domain Sensing of Targets Buried Under a Gaussian, Exponential, or Fractal Rough Interface
}

\author{
Traian Dogaru and Lawrence Carin, Fellow, IEEE
}

\begin{abstract}
We numerically examine subsurface sensing via an ultrawideband ground penetrating radar (GPR) system. The target is assumed to reside under a randomly rough air-ground interface and is illuminated by a pulsed plane wave. The underlying wave physics is addressed through application of the multiresolution time-domain (MRTD) algorithm. The scattered time-domain fields are parametrized as a random process and an optimal detection scheme is formulated, accounting for the clutter and target signature statistics. Detector performance is evaluated via receiver operating characteristics (ROCs) for variable sensor parameters (polarization and incident angle) and for several rough-surface statistical models.
\end{abstract}

Index Terms-Detection, radar, sensing, time domain.

\section{INTRODUCTION}

$\mathbf{U}$ LTRAWIDEBAND (UWB) radar techniques have been applied successfully to a broad range of problems in target detection, classification, and imaging. Of particular interest here, UWB radar has been employed in the context of short-pulse sensing of subsurface targets [1]. In this paper, we simulate such a system, by analyzing the time-domain fields scattered from a target buried under a rough air-ground interface. The time-domain scattered fields are processed via an optimal detector, from which we investigate the effects of surface roughness on the detection of subsurface targets.

The statistical analysis of time-domain electromagnetic scattering from a rough surface has been investigated previously [2], [3]. However, the vast majority of work on rough-surface scattering has been addressed in the frequency domain [4]-[7]. Moreover, previously, most such work has addressed the rough-surface scattering problem alone, without consideration of scattering from a target buried under such a surface. Motivated by new UWB systems that operate in the time domain [1], here we concentrate on time-domain scattering from a rough surface, and in particularon subsurface sensing in such an environment. Recently, there has been significant interest in the development of time-domain electromagnetic models, such as the finite-difference time-domain (FDTD) [8] and, more recently, the multiresolution time-domain (MRTD) [9], [10]. The latter has been employed in modeling rough surface scattering problems [10], wherein its advantages vis-à-vis the FDTD scheme have been discussed. Motivated by the attractiveness of the MRTD for rough-surface scattering, it is applied here for all electromagnetic simulations.

It is demonstrated that a Bayesian detector, explicitly accounting for the statistical character of the buried-target

Manuscript received February 28, 2000; revised February 18, 2001.

The authors are with the Department of Electrical and Computer Engineering, Duke University, Durham, NC 27708-0291 USA.

Publisher Item Identifier S 0196-2892(01)05477-8. signature, often yields markedly improved detector performance $v i s-\grave{a}$-vis a matched filter. In practice, one would typically employ an approximate model to quantify the statistical characteristics of the target signature. In the work presented here, in which we investigate the fundamental phenomenological issues in such a detection scenario, the target-signature statistics are computed via our electromagnetic scattering model. In particular, we parametrize the statistics of the backscattered target signature numerically through consideration of multiple realizations of the rough surface, the latter dictated by a prescribed surface statistics. The scattered fields employed to test the performance of the detector are likewise computed via an electromagnetic model.

Before proceeding, we note that there has been previous work on the detection of targets under a rough surface. For example, Tsang and colleagues [12]-[15] have investigated the angular correlation function (ACF) to discriminate man-made targets from rough-surface clutter. In [12]-[15] the authors have addressed the three-dimensional (3-D) problem. The ACF exploits the statistical properties of a rough surface from multiple aspects (orientations) and across multiple frequency bands. The advantage of ACF is that it is applicable to a given target buried under a specific rough surface, and therefore it may be implemented in practice. In the work discussed here, we consider an optimal (Bayesian) detector for time-domain excitation. Such a detector requires an ensemble of scattered waveforms from a buried target, implying knowledge of the target signature under an ensemble of rough surfaces. This is therefore less practical than the ACF, requiring that the ensemble of scattered waveforms be measured or computed a priori. However, the Bayesian processor discussed here is an optimal detector and therefore, this study allows us to place bounds on the ultimate performance of detecting a target under a rough surface, against which more practical techniques such as ACF can be compared. In addition to addressing the detection problem per se, we also examine the degree to which the target signature is randomized by the rough surface. In particular, as noted above, even if the target identity is known, the randomization of the incident and transmitted fields by the rough surface cause the target signature to be stochastic. This effect is addressed for several rough surfaces, this issue being of importance for any detection scheme.

\section{MRTD AND ROUGH SURFACE SCATTERING}

\section{A. MRTD Formulation}

We summarize the formulation of the MRTD algorithm for a two-dimensional (2-D) configuration using an expansion of the fields in terms of Haar scaling functions and a single level of 


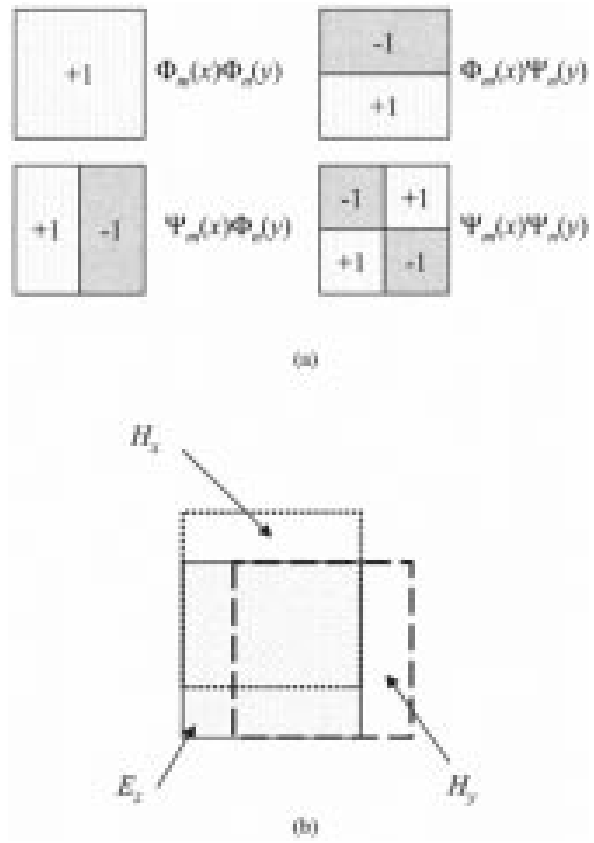

Fig. 1. (a) Prototype 2-D Haar scaling and wavelet functions and (b) support of the basis functions in the electric and magnetic field expansions using one level of Haar wavelets (two dimensions, TE polarization).

wavelets [16]. The model can be applied to an arbitrary number of Haar wavelet levels [17] at the cost of increased complexity. To simplify the presentation, we consider only a single wavelet level, this typically sufficient for scattering problems of interest. The MRTD is not limited to Haar wavelets, although algorithm complexity increases with the complexity of the underlying wavelet basis functions.

Fig. 1(a) shows the prototype Haar basis functions in the 2-D case. They are obtained from combinations of one-dimensional (1-D) Haar scaling and first-level wavelet functions $\Phi$ and $\Psi$, respectively. We consider a uniform grid with step $\Delta x$ in both spatial directions. The prototype Haar basis functions are shifted by an integer number of $\Delta x$ in both directions in order to cover the entire computational domain. For time discretization rectangular pulse functions are used, with a temporal step $\Delta t$ (equivalent to the Yee temporal discretization scheme). Fig. 1(b) represents the relative position of the basis-function support for the three components of a $T E$ polarized wave (the $T M$ case is similar if one interchanges the $E$ and $H$ components).

The field components are expanded using the basis described previously. At each cell of the computational grid and each time step, there are four coefficients per field component (corresponding to the combinations $\Phi_{x} \Phi_{y}, \Phi_{x} \Psi_{y}, \Psi_{x} \Phi_{y}, \Psi_{x} \Psi_{y}$ ). Maxwell's equations are sampled in time and space using a Galerkin procedure [18]. The result is a set of equations relating the set of field coefficients, which are updated every time step. A detailed description of the sampling procedure and the resulting equations in two dimensions is given in [17].

It can be demonstrated that the Haar-MRTD scheme, using scaling functions and one wavelet level, leads to a formulation equivalent to the Yee FDTD algorithm applied on a grid with half the cell size [17]. However, the MRTD formulation has the advantage that it can employ Haar wavelets in regions where high resolution is required (equivalent to FDTD at a sampling rate of $\Delta x / 2$ ), and scaling functions alone in regions where coarser spatial sampling is sufficient (equivalent to FDTD at a sampling rate of $\Delta x$ ). In other words, we obtain a self-consistent procedure for multiscale spatial gridding. Further wavelet levels can be added in certain regions, leading to an effective resolution increase in those regions of $2^{n}$ (where $n$ is the maximum wavelet level employed by the scheme).

\section{B. Modeling Rough Dielectric Interfaces with MRTD}

In addition to the aforementioned multiresolution properties of MRTD, another advantage of this model vis-a-vis the classic FDTD Yee scheme becomes apparent when one models media interfaces that do not conform to the computational grid. It is well known that the classical FDTD Yee scheme typically relies on a staircase approximation of such an interface. This approximation may introduce errors, especially when essential features of the scatterer are only a fraction of a cell dimension. Several techniques have been developed in the context of the FDTD algorithm to alleviate this problem [8]. The MRTD formulation allows a self-consistent treatment of interfaces of arbitrary shape [9], [10]. This becomes particularly useful for modeling scattering by a rough interface dividing two dielectric media. In a previous paper [10], we demonstrated that the MRTD scheme increases the accuracy of the rough surface scattering model as compared to the staircase FDTD model, assuming analogous spatial sampling rates. Although the errors introduced by the staircase FDTD model may be acceptable for surfaces with relatively "mild" roughness, the more accurate MRTD model may be necessary when the roughness is increased.

In our formulation, the dielectrics can be lossy, with the material properties ( $\varepsilon$ and $\sigma$ ) independent of frequency. Dispersive materials can be handled in a manner analogous to that applied to the FDTD [8]. Because of the presence of arbitrary inhomogeneity $\varepsilon(x, y)$ and $\sigma(x, y)$, the usual MRTD discretization procedure yields cross-terms between the $\Phi$ and $\Psi$ components of the electric field. Thus, the equations that update the various coefficients in the electric field expansion, at the cell $(m, n)$, become coupled, and they can be expressed as a matrix-vector equation for the field coefficients at that cell [9], [10]. The matrix involves the average permittivity and conductivity within cell $(m, n)$, weighted by the appropriate scaling/wavelet functions. If the medium is homogeneous inside one cell, the matrix is diagonal and the equations for that particular cell are decoupled. Consequently, the special matrix-vector equations need to be solved only for cells at the boundaries of two or more media.

The usual model for a 2-D rough surface involves a function $y=y(x)$, representing one realization of a random surface of particular statistics. The continuous function $y(x)$ is discretized (sampled) in the $x$ direction and, between the discretization points, it is approximated by straight segments. Fig. 2 shows a small portion of an arbitrary interface and the two models: MRTD (dashed line) and staircase (interface between the shaded and blank areas). While in the staircase FDTD case the material properties are generally sampled at the same rate as the fields (i.e., one grid cell), in the MRTD scheme the surface can be sampled more finely than the rate at which the fields are sampled. 


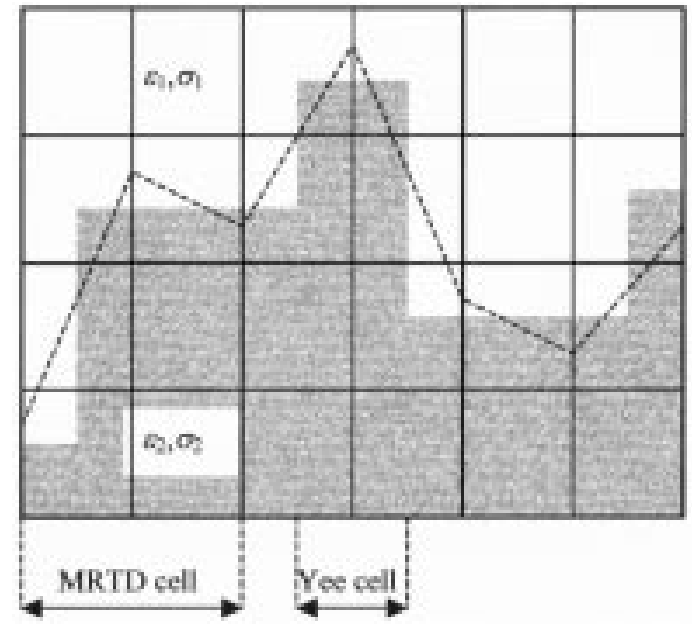

Fig. 2. Detail on the MRTD and staircase FDTD models of a rough surface (two dimensions, $T E$ polarization).

The typical FDTD sampling rate $\Delta x$, normally dictated by the shortest wavelength in the electromagnetic spectrum, may not be small enough for representing $y(x)$ without aliasing. Therefore, for rough-surface scattering computations, the FDTD $\Delta x$ must be made small enough to represent the rough surface well. The uniform gridding of the FDTD implies that this same spatial discretization must be used throughout, generally yielding over sampling away from the surface. With the MRTD algorithm, the rough interface can be sampled at a rate in excess of that used to represent the fields. The result is that, for the MRTD algorithm, the cell size is dictated by the shortest wavelength in the electromagnetic spectrum, and not by the finest feature of material inhomogeneity. Wavelets are added to the scaling functions to track fast field variations in the vicinity of the rough surface, with the fields away from the surface represented by scaling functions alone.

\section{ROUGH SURFACE MODELS}

\section{A. Traditional Models}

As mentioned in the previous section, the interface between the two-dielectric media is modeled as a random process with particular statistical parameters. We restrict our model to the 2-D case. For the "traditional" random processes considered in this section, the samples $y(x)$ are assumed to have a Gaussian probability density function. Scattering from a surface with an exponential density function (non-Gaussian) has been discussed in [13]. The most widely applied type of rough surface model is characterized by a Gaussian autocorrelation function [5]. For a surface with variance $\sigma^{2}$, the autocorrelation is expressed as

$$
R(\Delta x)=E[y(x) y(x+\Delta x)]=\sigma^{2} \exp \left(-\frac{\Delta x^{2}}{l^{2}}\right)
$$

with the parameter $l$ termed the correlation length. Another frequently employed autocorrelation is the exponential function. This correlation function has generally been avoided in analytic studies of scattering from rough surface, because it is not differentiable at the origin. Since we are concerned with the numerical analysis of rough-surface scattering, this does not present an obstacle in our study. The exponential correlation functions has the form

$$
R(\Delta x)=E[y(x) y(x+\Delta x)]=\sigma^{2} \exp \left(-\frac{|\Delta x|}{L}\right) .
$$

Analogous to the Gaussian case, the parameter $L$ represents the correlation length. Authors have proposed other models, constituting hybrids of the Gaussian and exponential functions, characterized by two or more correlation-length-type parameters, taking into account the roughness at different scales. Although the methods presented in this paper are applicable to hybrid surface models, we restrict ourselves to Gaussian and exponential surfaces.

In practice, the random process (surface) is generated in the Fourier domain, by passing a Gaussian white-noise process through a filter with a spatial-frequency response corresponding to the desired rough-surface spectrum [5].

\section{B. Bandlimited Fractal Models}

Although simple correlation functions like the Gaussian or the exponential are commonly used in many theoretical studies of rough-surface scattering, they are not necessarily representative of many categories of natural surfaces. In particular, natural scenes such as sea, forest and other vegetated regions present a multiscale structure that cannot be described by only one parameter (the correlation length). The recently developed fractal framework [19] has proven a better approach for describing a multitude of natural phenomena, including natural rough interfaces. Recent papers have studied the scattering of electromagnetic waves from fractal surfaces, using analytical as well as numerical methods [20]-[22]. Theoretically, random fractal surface models take into account the roughness over an infinite range of scales. However, fractal surfaces cannot be handled rigorously in practice because of their peculiar mathematical properties, among which are nonstationarity and the consequent inappropriateness of defining a power spectrum. For practical purposes we therefore must restrict ourselves to the study of bandlimited fractals [20]. The finite length of the sequence is equivalent to considering a lower limit of the frequency spectrum, whereas sampling at finite intervals truncates the spectrum at high frequencies. In fact, any measurement process would present similar limitations, therefore making the bandlimited fractal a realistic model.

In the following, we consider the bandlimited Weierstrass process [20]-[23]:

$$
\begin{aligned}
w(x) & =\sigma \sqrt{\frac{2\left(1-b^{-2 H}\right)}{\left.b^{-2 H N_{1}}-b^{-2 H\left(N_{2}+1\right.}\right)}} \\
& \cdot \sum_{n=-N_{1}}^{N_{2}} b^{-H n} \cos \left(2 \pi b^{n} x+\varphi_{n}\right)
\end{aligned}
$$

where

$\sigma \quad$ standard deviation;

$H \quad$ Hurst exponent with values between 0 and 1;

$b>1 \quad$ constant (we take $b=\sqrt{\pi}$ );

$\varphi_{n} \quad$ random phases, uniformly distributed between 0 and $2 \pi$. 


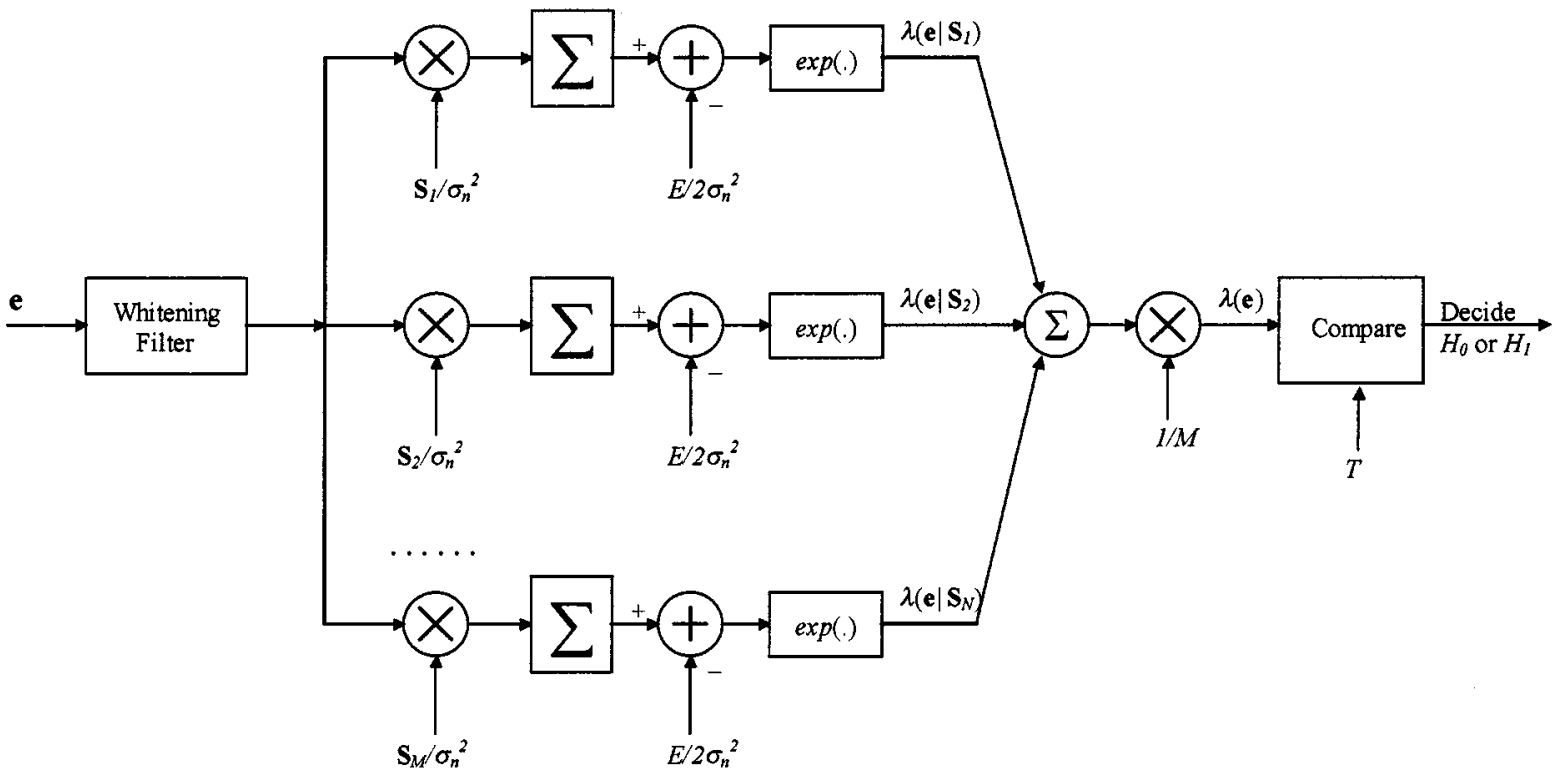

Fig. 3. Optimal detector for a random signal in additive clutter, using $\mathrm{M}$ realizations of the random signature. $s_{1}, s_{2}, \ldots, s_{M}$ are $M$ independent realizations of the target signature, $E$ is the average target signature energy, and $\sigma_{n}$ is the clutter variance. Each channel computes the likelihood ratio for one given realization of the target signature.

The fractal dimension of this process is $D=2-H$. It can be shown that the spectrum of a Weierstrass process follows a power law [i.e., $S(k) \propto k^{-2 H-1}$ ] [20]-[23].

The integers $N_{1}$ and $N_{2}$ in (3) represent the lowest and the highest harmonics in the spectrum. In a pure fractal process, we should have $N_{1}=-\infty$ and $N_{2}=\infty$, such that the frequency runs from 0 to $\infty$. However, in this case, the amplitude of the zero-frequency (dc) component would go to infinity, so we must keep $N_{1}$ finite. Also, the sampling process requires $N_{2}$ to be finite. One constraint in choosing these integers is to include enough discrete spectral modes such that the highest-frequency component has small amplitude compared to the lowest-frequency component. We notice that the amplitude decay is reduced as $H$ decreases (higher fractal dimension), thus requiring more spectral modes to be taken into account. On the other hand, this increases the maximum frequency in the spectrum, putting another constraint on the discretization rate (one should notice that this tradeoff is independent of $b$ ). Summarizing, for higher fractal dimensions (close to 2), it is difficult to obtain a good representation of the Weierstrass process, unless we consider very fine discretization rates. Therefore, in this paper, we limit ourselves to the study of surfaces with fractal dimension less than or equal to 1.5 .

It should be noted that the value of $N_{1}$ is extremely important in characterizing the statistics of both the rough surface and the associated scattered fields. In particular, changing $N_{1}$ for a set of surfaces leads to significant variation in the energy of the fields scattered from those surfaces. The parameter $N_{1}$ should be taken greater (or at least equal) to zero, otherwise the surface would include less than a period of the lowest frequency component (which has the largest amplitude). Since the phase of this component is random, it is possible to obtain a surface average other than zero, which is undesirable. It is not difficult to see that the larger $N_{1}$, the smaller the energy of the return in the backscatter direction (which is of interest in our problem), because the large-scale features in the rough surface (corresponding to the lower frequencies), are eliminated. It is also worth mentioning that, although a pure Weierstrass fractal surface is nonstationary [19], its bandlimited version is stationary and its PSD can be computed in closed-form [20]. We have found that the fields scattered by such a surface are stationary as well. This is important for the detector design (see Section IV), where one of the basic assumptions is the stationarity of the clutter.

\section{Truncating the Surface}

In any numerical model of rough surface scattering, the surface must be finite, making edge effects an important issue. One solution to this problem is to consider illumination by a beam [4]-[7]. This is often employed as the incident field in frequency-domain integral-equation solvers [4]-[6]. However, the beam approach is difficult to implement with time-domain methods, if one tries to model wideband electromagnetic scattering. This is because the beam width increases with the wavelength, which means that for the low frequencies of the spectrum we need an impractically large computational domain. In this paper, we are interested in the time-domain statistical characterization of the fields scattered by a rough surface. For this purpose, we consider scattering from a finite-extent surface, under pulsed plane-wave excitation. As mentioned, the plane-wave excitation gives rise to edge effects at the ends of the finite rough surface. The received time sequence is windowed temporally, with the scattered-field statistics computed by considering only a portion of the transient signature in the middle of the time-domain response, this weakly affected by edge diffraction (in the beam approach, the beam width windows the problem spatially). We also emphasize that, as indicated in Fig. 4, the MRTD model considers a finite 2-D rough surface in the vicinity of an infinite dielectric half space (the latter accounted for by the con- 


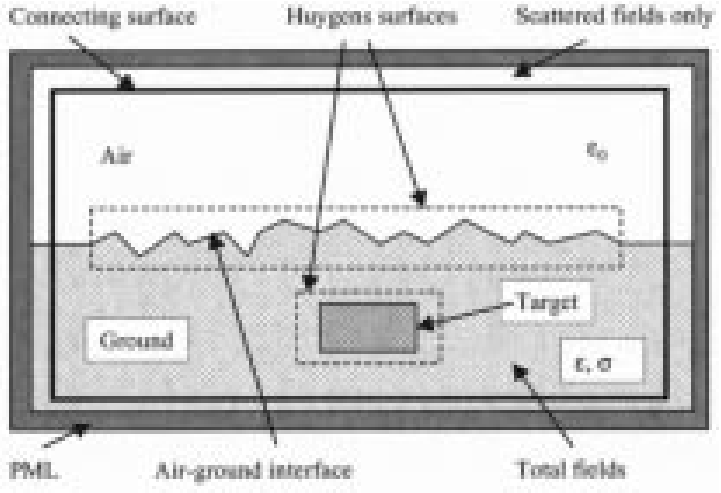

(a)

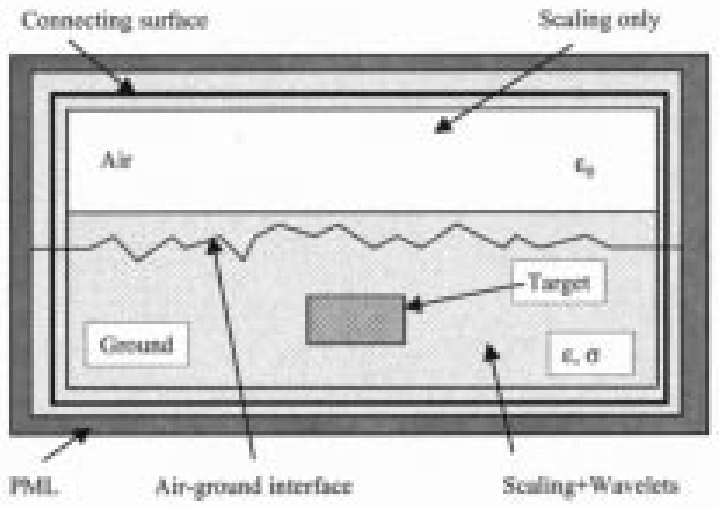

(b)

Fig. 4. MRTD computational domain. (a) Incident field implementation, PML, and near-to-far zone transformation and (b) repartition of scaling and wavelet basis functions.

necting surface that gives rise to the half-space plane-wave excitation). The diffracted fields from the ends of the rough surface are therefore generated by the relatively smooth transition from a half space to a rough surface. Moreover, the rough surface is tapered at the ends, to avoid sharp peaks in the vicinity of the terminal points.

\section{OPtimal Detection of Buried TARgets}

The problem of interest is detection of a buried target in the presence of a rough interface. There are two signals involved in the detection scheme: the target response (which is the signal we wish to detect) and the fields scattered by the interface (clutter). In the following, the various sampled signals are considered as vectors in an $N$-dimensional signal space, where $\mathbf{e}=\left[e_{1} e_{2} \cdots e_{N}\right]^{T}$ represents the received signal, $\mathbf{c}=\left[c_{1} c_{2} \cdots c_{N}\right]^{T}$ represents the random clutter response, and $\mathbf{s}=\left[s_{1} s_{2} \cdots s_{N}\right]^{T}$ represents the target response (assuming time-domain scattered waveforms with $N$ temporal samples). At the detector output, we must decide between hypothesis $H_{0}$, in which $\mathbf{e}=\mathbf{c}$ (the received signal consists of clutter alone) and hypothesis $H_{1}$, in which $\mathbf{e}=\mathbf{s}+\mathbf{c}$ (the target is present).

We assume that the clutter is a Gaussian, wide sense stationary and uncorrelated random process. In numerical experiments, we have verified that the first two assumptions are valid

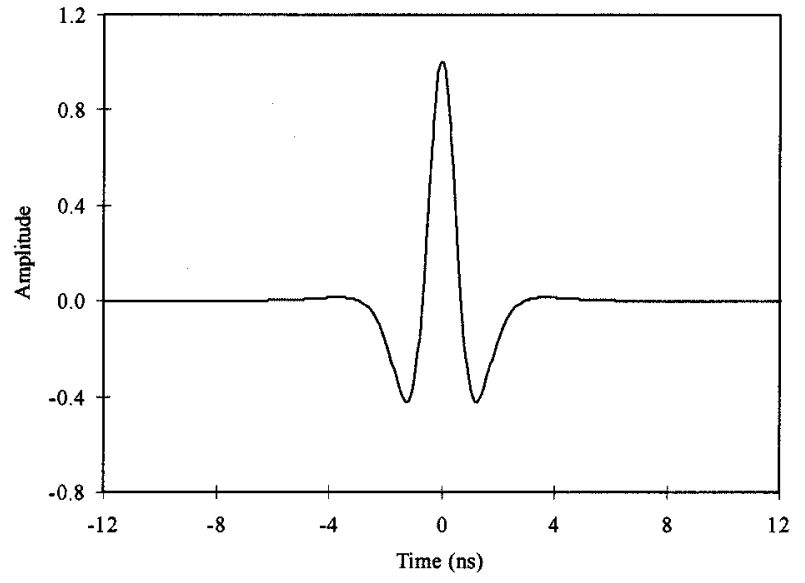

(a)

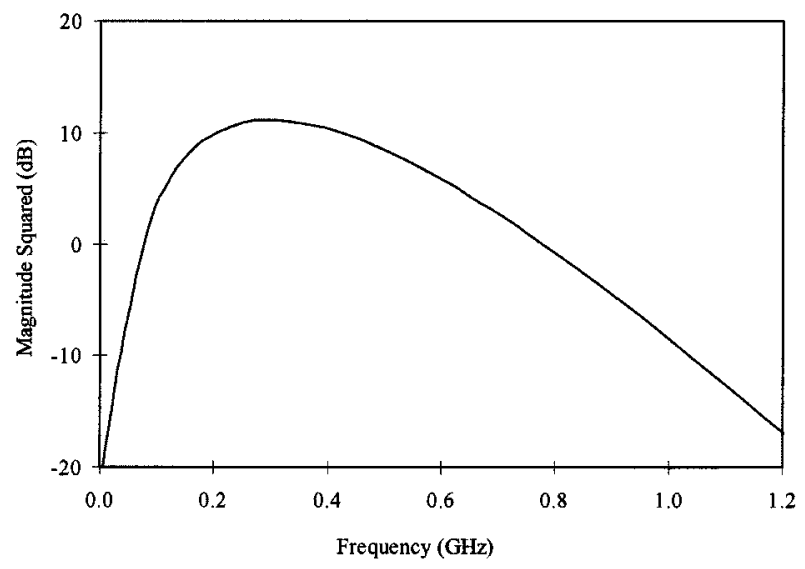

(b)

Fig. 5. Incident pulse (Rayleigh, fourth order) in (a) time and (b) frequency domain.

for the rough surfaces modeled here. However, the fields scattered by a correlated random surface are correlated themselves. In order to satisfy the third assumption, we precede the detector by a whitening filter [11].

It is important to recognize that the target signature $\mathbf{s}$ is a random process, even if the target identity and depth are assumed known. This is manifested because the fields transmitted through the rough interface are stochastic, due to transmission through a randomly rough surface. Similar stochastic distortion occurs when the fields scattered by the target travel from the ground into the air through the rough interface. The stochastic character of $\mathbf{s}$ has important implications in the detector design, because a simple matched filter is suboptimal in such a situation [11]. In particular, if the target signature $\mathbf{s}$ is deterministic, the optimal detector is the likelihood-ratio test

$$
\Lambda(\mathbf{e})=\frac{\mathrm{p}_{\mathbf{c}}(\mathbf{e}-\mathbf{s})}{\mathrm{p}_{\mathbf{c}}(\mathbf{e})} \stackrel{H_{1}}{\gtrless} T
$$

where $\mathrm{p}_{c}(\mathbf{c})$ represents the distribution of the clutter. After employing the aforementioned whitening filter $\mathbf{c}$ is an uncorrelated 


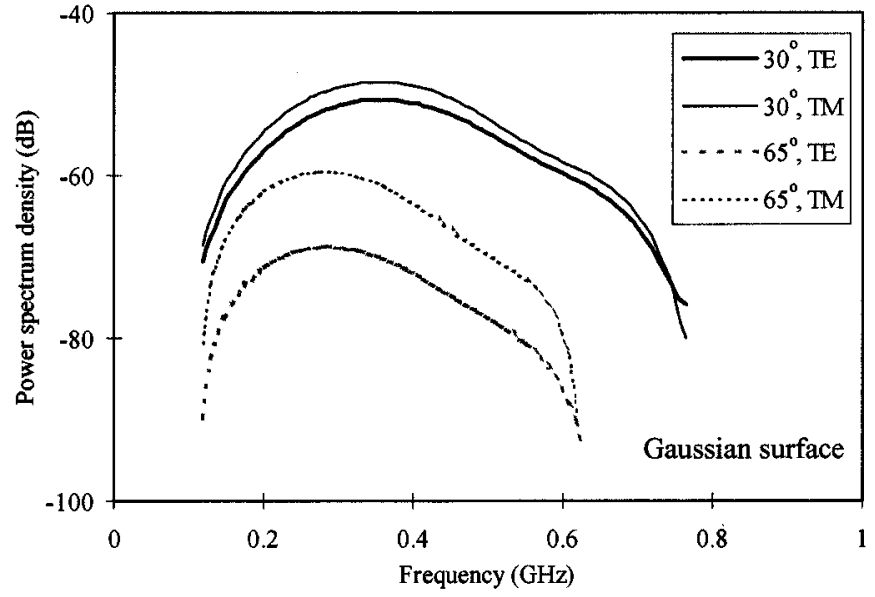

Fig. 6. Power spectrum density produced by a Gaussian surface with $\sigma=$ $3.95 \mathrm{~cm}$ and $L=25 \mathrm{~cm}$ for the excitation described in Fig. 5 .

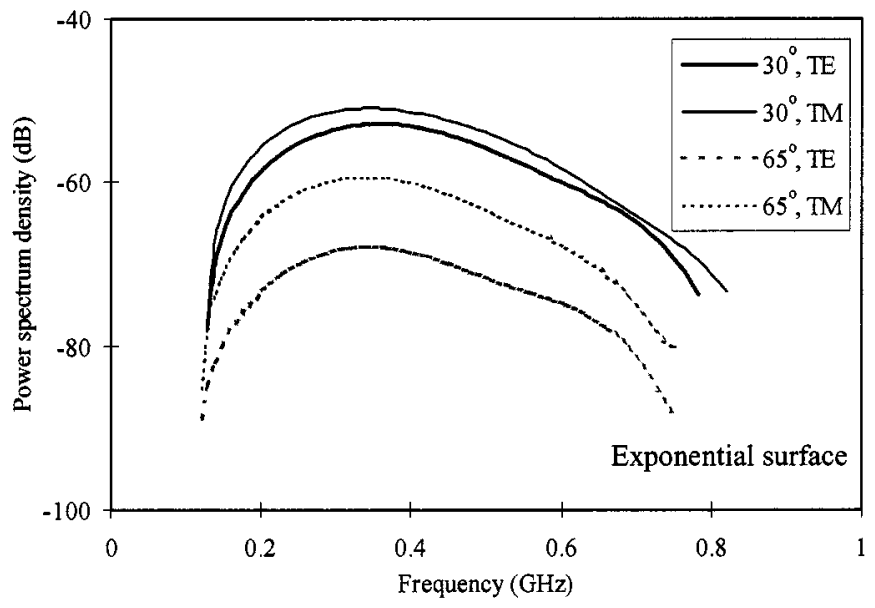

Fig. 7. Power spectrum density produced by an exponential surface with $\sigma=$ $3.95 \mathrm{~cm}$ and $L=25 \mathrm{~cm}$ for the excitation described in Fig. 5.

Gaussian process, for which the likelihood ratio in (4) reduces to a form that is implemented as a matched filter. The threshold $T$ is varied to generate the receiver operating characteristic (ROC) [11].

One can model the randomness in the target response by introducing a generalized stochastic parameter $\theta$. Instead of assuming the target signature $\mathbf{s}$ is known exactly, we introduce an uncertainty, which we symbolize by the notation $\mathbf{s}(\theta)$, where $\theta$ represents a generalized vector of stochastic parameters responsible for the random nature of $\mathbf{s}$. Thus, under the hypothesis $H_{1}$, the received signal becomes $\mathbf{e}=\mathbf{s}(\theta)+\mathbf{c}$. The simple likelihood ratio in (4) is now generalized as

$$
\Lambda(\mathbf{e})=\frac{\int \mathrm{p}_{\mathbf{c}}(\mathbf{e}-\mathbf{s}(\theta)) d \theta}{\mathrm{p}_{\mathbf{c}}(\mathbf{e})}=\int \Lambda(\mathbf{e} \mid \theta) \mathrm{p}_{\grave{e}}(\theta) d \theta \underset{H_{0}}{\stackrel{H_{1}}{\gtrless}} T
$$

where $\Lambda(\mathbf{e} \mid \theta)$ represents the likelihood ratio for a particular $\mathbf{s}(\theta)$, and $\mathrm{p}_{\theta}(\theta)$ is the probability density function (pdf) of the vector $\theta$.

Note that we have not specified $\mathrm{p}_{\theta}(\theta)$, nor do we quantify its distribution. Although one could attempt to model $\theta$ as a physical quantity, we avoid this and consider an alternative manner

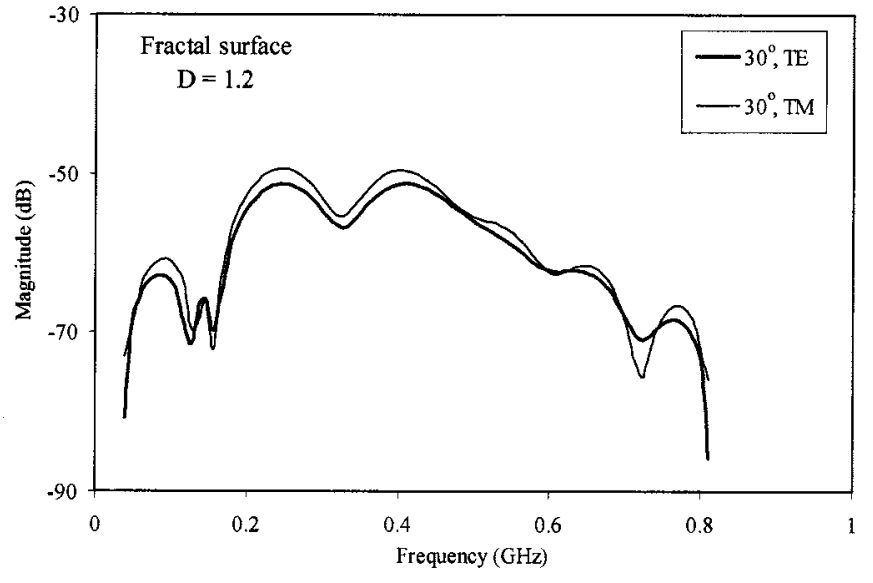

(a)

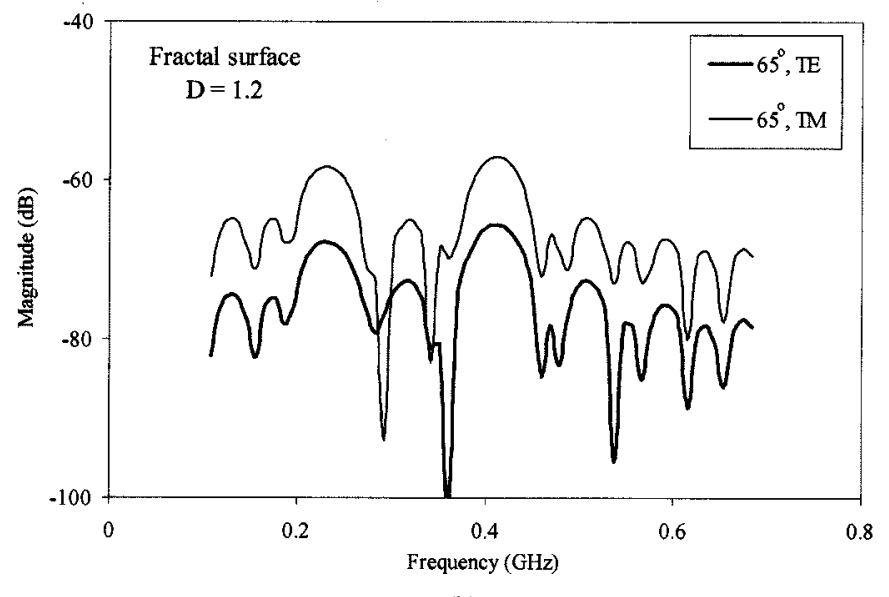

(b)

Fig. 8. Power spectrum density produced by a fractal surface with $\sigma=6.25$ $\mathrm{cm}$ and $D=1.2$ for the excitation described in Fig. 5. (a) Incidence at $30^{\circ}$ and (b) incidence at $65^{\circ}$.

of computing the likelihood ratio. Considering (5), note that the expression on the right side is simply the ensemble average of $\Lambda(\mathbf{e} \mid \theta)$, computed for all possible values of $\theta$. We can approximate this quantity numerically by performing Monte Carlo integration. Thus, we consider $M$ realizations of the random vector $\theta$ (physically corresponding to $M$ realizations of the rough surface), the $m$ th of which is represented by $\theta_{m}$ and compute the approximate likelihood ratio as

$$
\Lambda(\mathbf{e})=\int \Lambda(\mathbf{e} \mid \theta) \mathrm{p}_{\grave{e}}(\theta) d \theta \approx \frac{1}{M} \sum_{m=1}^{M} \Lambda\left(\mathbf{e} \mid \theta_{m}\right) \underset{H_{0}}{\stackrel{H_{1}}{\gtrless}} T .
$$

Consequently, we obtain the structure of the optimal receiver described in Fig. 3. The number of projections $M$ is determined empirically by studying the convergence of the detector output as a function of $M$. In all the simulations presented in the next section, 40-50 projections were typically sufficient for convergence [3]. The ensemble of target scattered waveforms $\mathbf{s}\left(\theta_{m}\right)$ are computed via our MRTD scattering model, for the target of interest under $M$ realizations of the rough surface, the latter described by a prescribed statistical model. 


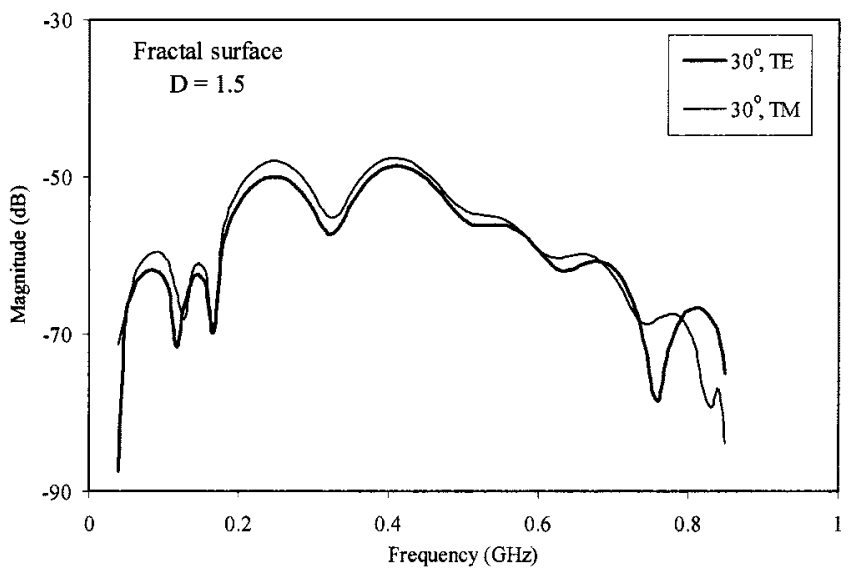

(a)

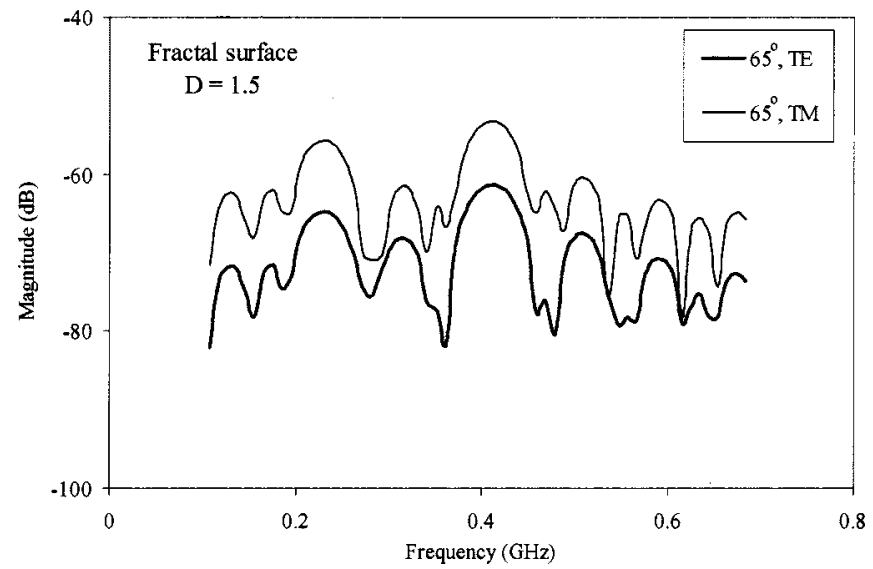

(b)

Fig. 9. Power spectrum density produced by a fractal surface with $\sigma=6.25$ $\mathrm{cm}$ and $D=1.5$, for the excitation described in Fig. 5. (a) incidence at $30^{\circ}$ and (b) incidence at $65^{\circ}$.

\section{RESULTS}

\section{A. Implementation}

We consider short-pulse electromagnetic scattering from a dielectric target buried under a rough air-ground interface. The clutter statistics are computed by considering time-domain electromagnetic scattering from the rough surface, in the absence of a subsurface target. The clutter statistics are employed in the design of the whitening filter. Moreover, as discussed above, the model is used to compute the ensemble of stochastic target signatures $\mathbf{s}\left(\theta_{m}\right)$ characteristic of the target buried under the rough surface. Each realization of the target signature $\mathbf{s}\left(\theta_{m}\right)$ is computed by considering the target under a particular realization of the rough surface. We also compute the scattered fields from the same surface, in the absence of the target. By subtracting the latter from the former, we yield one realization of the target signature. To test the detector, we generate a set of stochastic scattered waveforms when the target is absent (hypothesis $H_{0}$ ) and when it is present (hypothesis $H_{1}$ ), and variation of the detector threshold $T$ yields the detector performance in the form of ROC. In order to obtain meaningful statistical data, we consider many realizations of the rough surface for a given set of parameters.

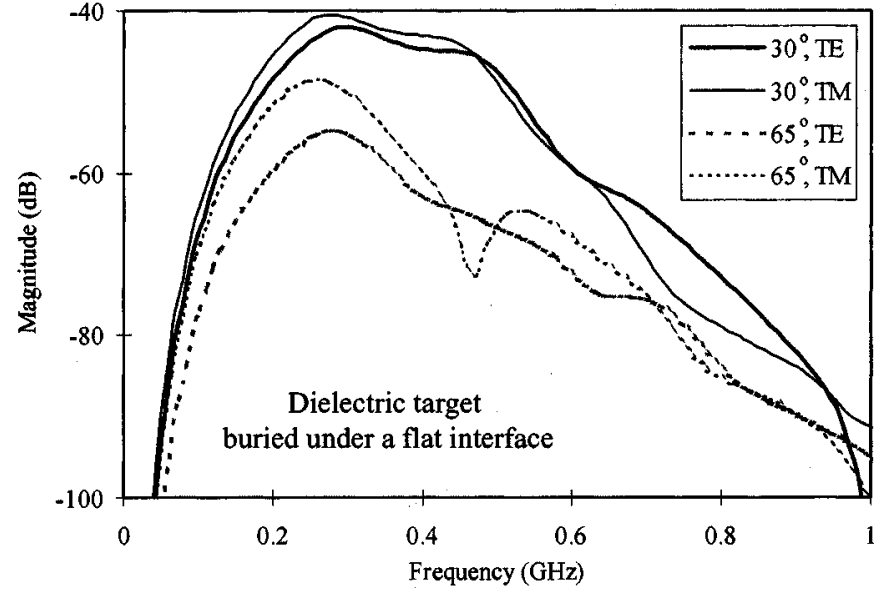

Fig. 10. Energy spectrum density for a $37.5 \times 12.5 \mathrm{~cm}$ dielectric target with $\varepsilon_{r}=2$, buried $25 \mathrm{~cm}$ beneath a flat air-ground interface. The soil has $\varepsilon_{r}=6$ and $\sigma=0.005 \mathrm{~S} / \mathrm{m}$. The excitation is described in Fig. 5 .

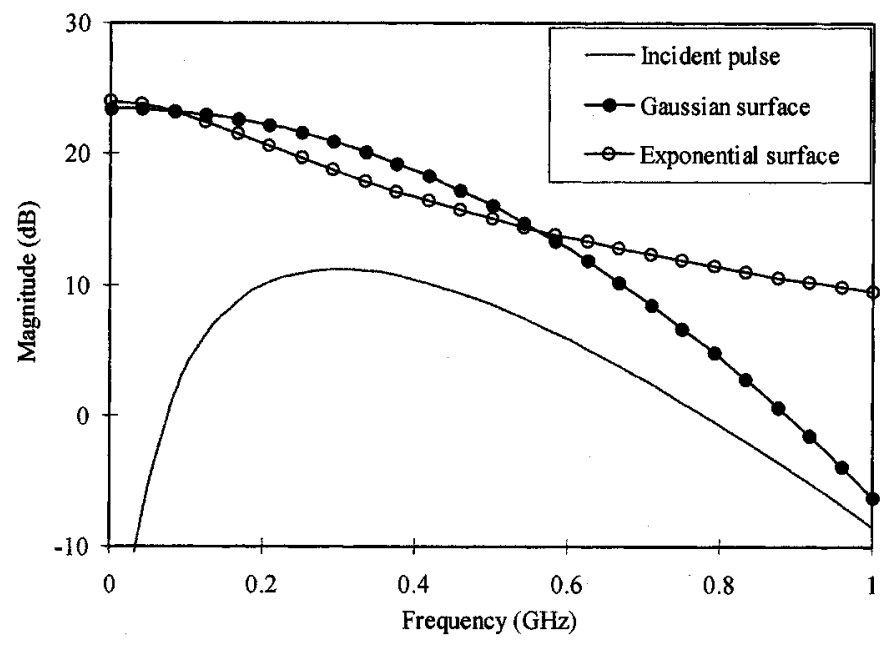

Fig. 11. Comparison between the incident pulse spectrum $\left(\lambda_{c}=1 \mathrm{~m}\right)$ and the rough-surface power spectrum density. The Gaussian and exponential surfaces have $L=0.25 \mathrm{~m}$.

Distinct computations are used to compute the clutter statistics, the ensemble of $\mathbf{s}\left(\theta_{m}\right)$, as well as the waveforms employed to generate the ROCs (we do not test and train on the same data).

The large number of rough-surface calculations (typically 600 rough-surface calculations are used in training and testing the detector) requires an efficient implementation of the MRTD algorithm. A schematic drawing of the MRTD computational domain is shown in Fig. 4. The soil is modeled as a lossy dielectric, with $\varepsilon_{r}=6$ and $\sigma=0.005 \mathrm{~S} / \mathrm{m}$, independent of frequency. These parameters are typical for dry soil. A more realistic soil model should account for a variation of these parameters with the frequency. However, for shallow targets, the propagation distance inside the soil (in the region of interest) is electrically very small, making the dispersion effects negligible. The 2-D target is modeled as a rectangular cylinder (infinite in the transverse direction), with dimensions $37.5 \mathrm{~cm} \times 12.5 \mathrm{~cm}$, composed of a lossless dielectric with $\varepsilon_{r}=2$. The target is buried at a depth of $25 \mathrm{~cm}$ (measured from the average position of the rough interface to the top of the target). Alternative targets could be considered, but the 


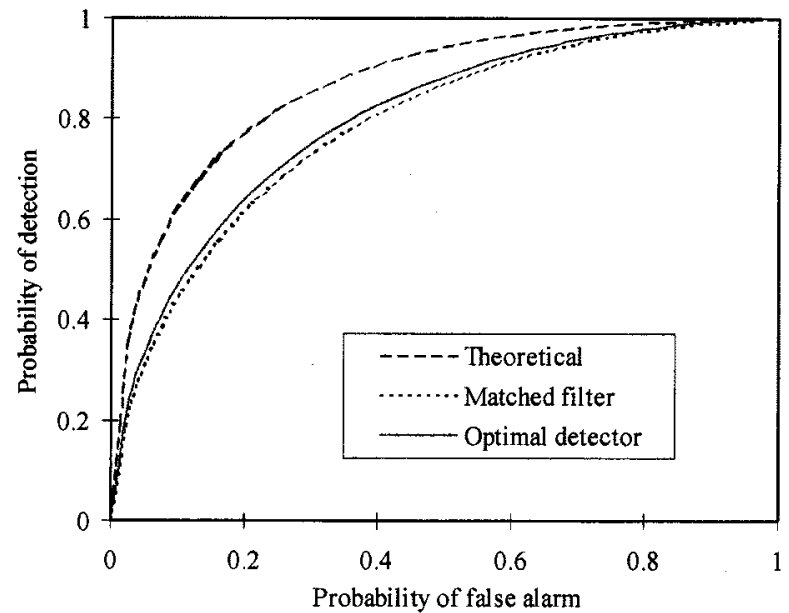

(a)

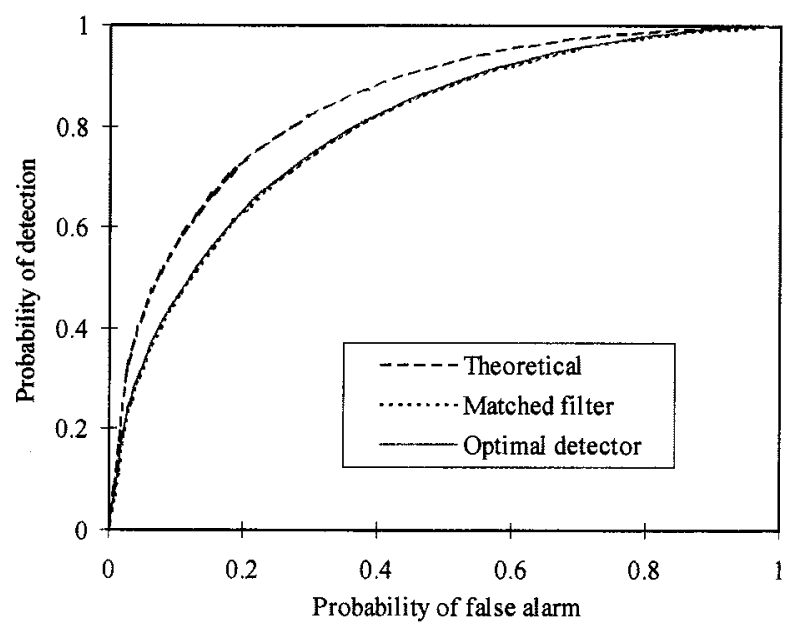

(b)

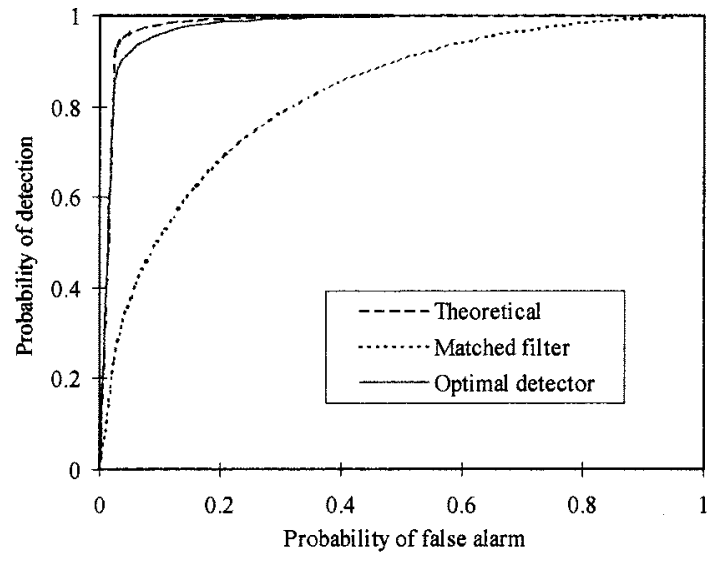

(a)

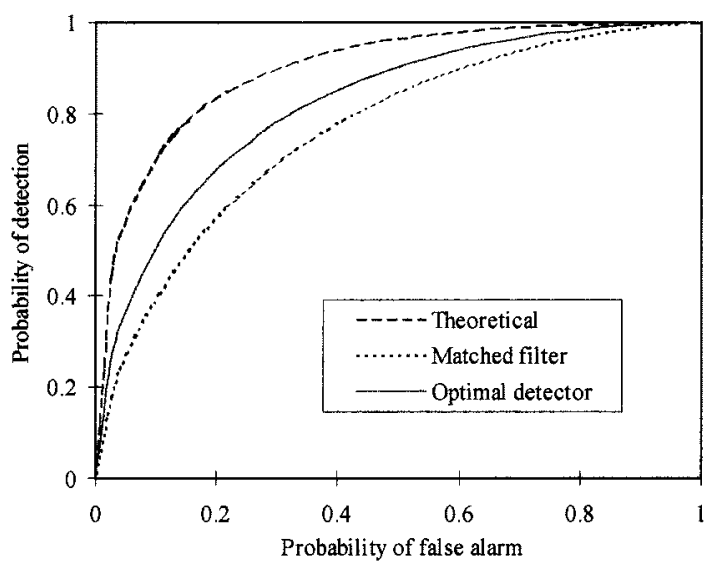

(b)

Fig. 13. Receiver operating characteristics for a Gaussian surface with $\sigma=$ $3.95 \mathrm{~cm}$ and $L=25 \mathrm{~cm}$, incidence at $65^{\circ}$. (a) $T E$ polarization and (b) $T M$ polarization.

Fig. 12. Receiver operating characteristics for a Gaussian surface with $\sigma=$ $3.95 \mathrm{~cm}$ and $L=25 \mathrm{~cm}$, incidence at $30^{\circ}$. (a) $T E$ polarization and (b) $T M$ polarization.

focus here is on the impact of the rough-surface statistics on detection performance, and therefore the emphasis is placed on addressing several rough-surface statistical models.

The incident field is implemented as a pulsed plane wave, radiating in the presence of an infinite planar half space. The rough surface and target constitute perturbations to this incident wave, and yield the scattered fields. The incident time-domain waveform and associated frequency spectrum are shown in Fig. 5. This type of pulse shape (Rayleigh fourth order [24]) is typical of ultrawideband synthetic aperture radar (SAR) systems, and its central frequency $(300 \mathrm{MHz})$ is also characteristic of applications involving subsurface sensing [1]. The discretization rate for the MRTD scheme is 40 Haar scaling functions per central wavelength in air, which implies about six Haar scaling functions per wavelength at the smallest wavelength in the spectrum, in the denser medium (when wavelets are considered as well,

this corresponds to 12 cells per smallest wavelength in terms of equivalent Yee cells). The observations are always made in backscatter at a distance of $1000 \lambda_{c}$ (far zone) from the target, where $\lambda_{c}$ is the central wavelength of the pulse in air.

Of interest is the length of the rough surface employed in the computations. As indicated in Fig. 4, the rough surface is modeled as a finite-length perturbation to an infinite half space. The onset of the rough surface is smoothed such that diffraction at the edge of the rough surface is minimized. Moreover, recall that we are only interested in the clutter signature $\mathbf{c}$ in the vicinity of the (time-limited) target signature s. Consequently, for calculation of the stochastic $\mathbf{e}, \mathbf{s}$ and $\mathbf{c}$, we only use the time-domain scattered fields for times, during which the incident pulsed plane wave is well separated temporally from diffraction at the rough-surface edges. To determine an appropriate rough-surface length, we compute the statistics of $\mathbf{s}$ and $\mathbf{c}$ for surface length $L$ and separately for a length larger than $L$. When we no longer see a change in the scattered-field statistics (e.g., the first few coefficients of the autocorrelation sequence), the surface $L$ is deemed long enough. For the cases studied here, we have found 


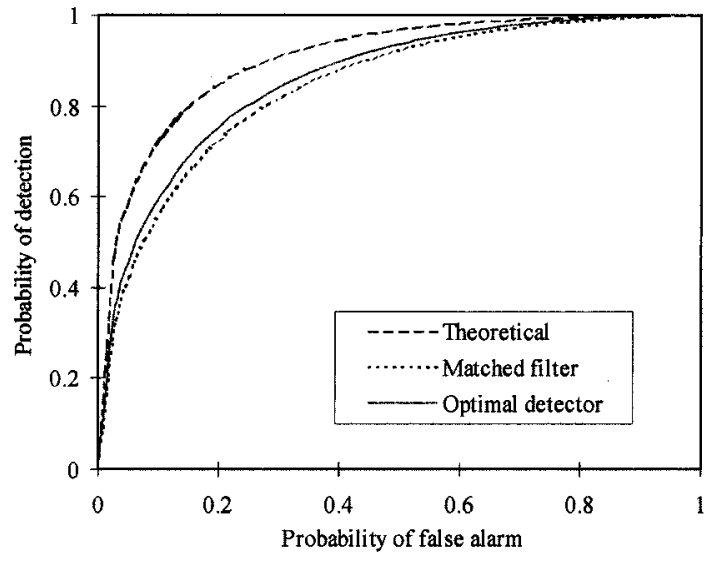

(a)

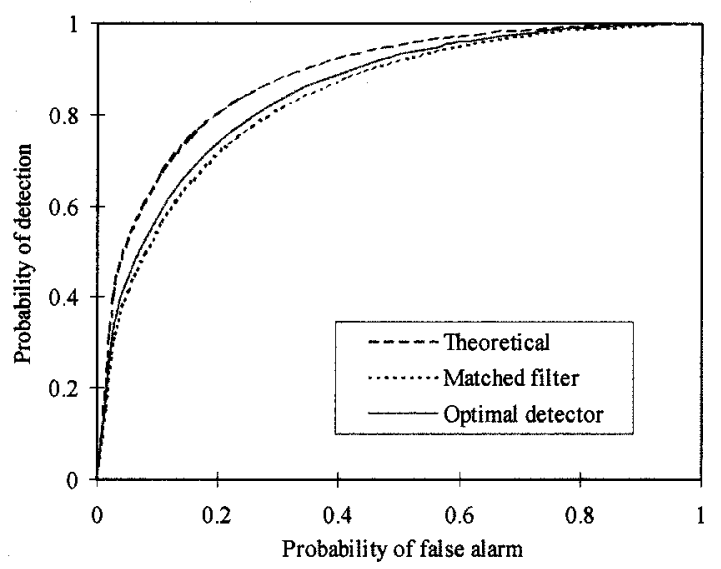

(b)

Fig. 14. Receiver operating characteristics for an exponential surface with $\sigma=3.95 \mathrm{~cm}$ and $L=25 \mathrm{~cm}$, incidence at $30^{\circ}$. (a) $T E$ polarization and (b) $T M$ polarization.

that a surface length of about $12.5 \lambda_{c}$ is normally sufficient, even at incidence angles of $65^{\circ}$. Numerical details on this issue can be found in [17].

\section{B. Spectral Estimation}

In Figs. 6-9, we plot the estimated power spectral density (PSD) of the clutter $\mathbf{c}$ for Gaussian, exponential, and Weierstrass fractal surfaces, at different angles of incidence, for both $T E$ and $T M$ polarization. The Gaussian (Fig. 6) and exponential (Fig. 7) surfaces under consideration have standard deviation $\sigma=3.95 \mathrm{~cm}$ and $25 \mathrm{~cm}$ correlation length, while the central wavelength of the incident pulse is $\lambda_{c}=1 \mathrm{~m}$. The fractal surfaces are characterized by the standard deviation $\sigma=6.25 \mathrm{~cm}$ and fractal dimension $D=1.2$ (Fig. 8) and $D=1.5$ (Fig. 9). Other important parameters of the Weierstrass fractal surfaces are the order of the lowest and highest harmonics, $N_{1}$ and $N_{2}$. For fractal dimension $D=1.2, N_{1}=2$ and $N_{2}=12$, while for fractal dimension $D=1.5, N_{1}=2$ and $N_{2}=15$.

We note that the fundamental character of the PSD for the Gaussian and exponential surfaces (Figs. 6 and 7) is markedly

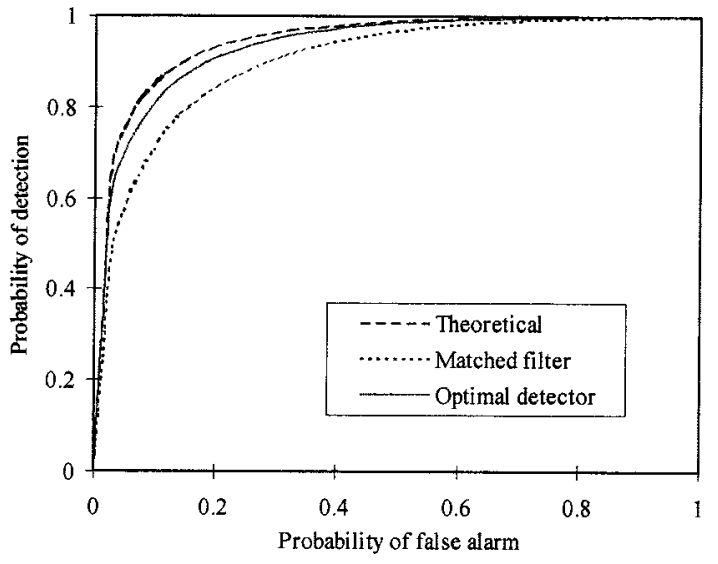

(a)

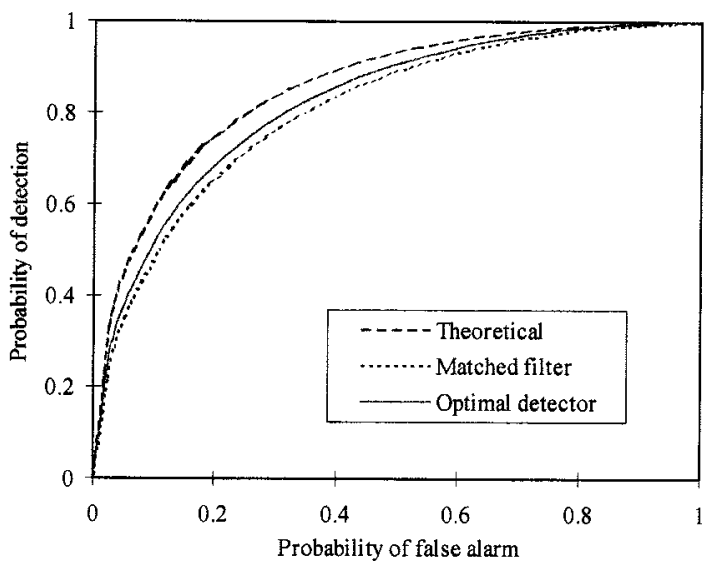

(b)

Fig. 15. Receiver operating characteristics for an exponential surface with $\sigma=3.95 \mathrm{~cm}$ and $L=25 \mathrm{~cm}$, incidence at $65^{\circ}$. (a) $T E$ polarization and (b) $T M$ polarization.

different from that of the fractal surfaces (Figs. 8 and 9). This is interpreted as follows. Considering the Weierstrass fractal surfaces described in (3), we note that these surfaces are characterized by the superposition of harmonics with random phase. Each harmonic corresponds to a finite periodic surface, and therefore, diffraction from each is characterized by scattering in terms of a set of Floquet modes [20]. Each Floquet mode propagates at a frequency-dependent angle, with the angle of propagation representative of the backscattered direction at particular frequencies. This yields the fractal-surface induced clutter in Figs. 8 and 9, in which particular Floquet modes yield a strong backscatter response at particular frequencies. The number of Floquet modes excited is dependent on the excitation incidence angle, increasing as one gets closer to grazing.

Detector performance will be dictated largely by the ratio of the target-signature energy to the clutter energy. It is therefore of interest to consider the frequency-dependent target signature. In Fig. 10, we plot the frequency dependence of the target signature for the incidence angles and polarizations addressed in Figs. 6-9, for a flat air-soil interface. We notice that the target signature decreases in strength as the incidence angle increases. 


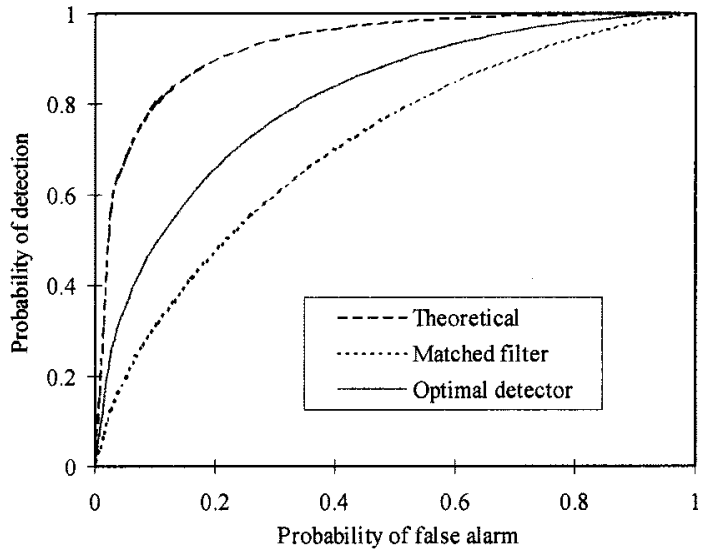

(a)

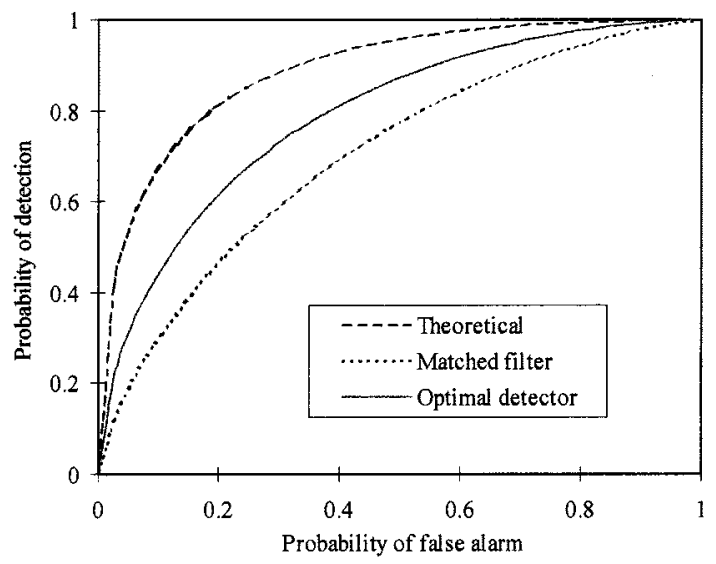

(b)

Fig. 16. Receiver operating characteristics for a fractal surface with $\sigma=6.25$ $\mathrm{cm}$ and $D=1.2$, incidence at $30^{\circ}$. (a) $T E$ polarization and (b) $T M$ polarization.

However, the clutter energy also decreases markedly with increasing incidence angle and as a result the target-to-clutter ratio is larger with increased incidence angle, thereby yielding improved detector performance with increasing incidence angle, as proved in the next section.

In Fig. 11, we plot the power spectral densities of the Gaussian and exponential surfaces considered here, as well as the incident-pulse spectrum. Note that the exponential surface has more high-frequency character, this manifested by more fine structure in the surface roughness. By contrast, at frequencies at which the incident pulse has maximum energy, the Gaussian surface is stronger. These curves will play an important role in analyzing the detector performance, in the next section.

\section{Detector Performance}

The detector performance is evaluated by plotting the ROC. This represents the variation of the probability of detection $P_{D}$ as a function of the probability of false alarm $P_{F}$ [by continuously varying the detector threshold $T$, see (4)-(6)]. All the

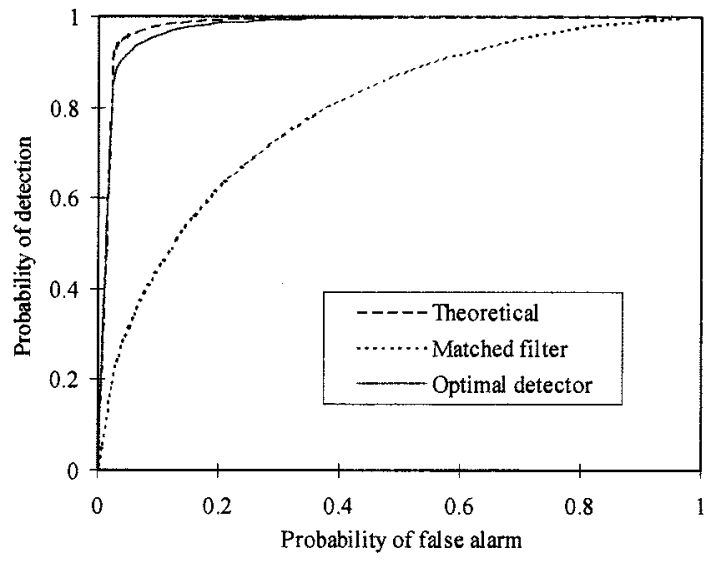

(a)

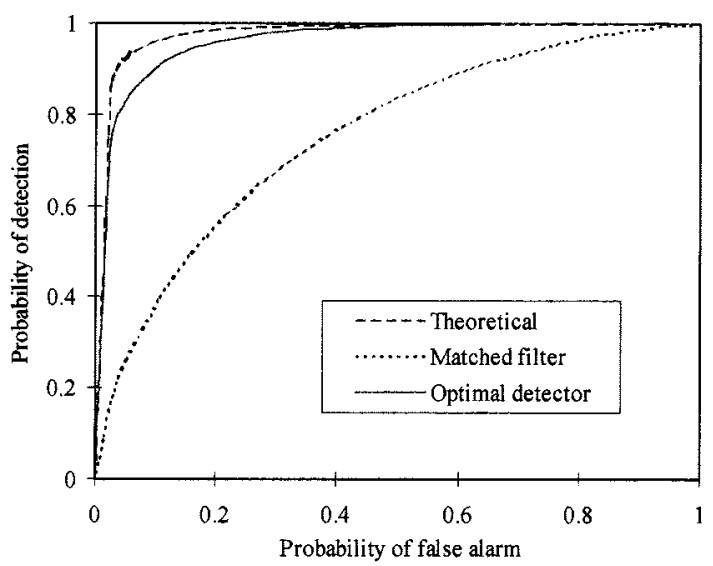

(b)

Fig. 17. Receiver operating characteristics for a fractal surface with $\sigma=6.25$ $\mathrm{cm}$ and $D=1.2$, incidence at $65^{\circ}$. (a) $T E$ polarization and (b) $T M$ polarization.

relevant target and surface parameters have been described in the previous two sections. We plot three curves on each graph. One represents the idealized matched filter performance. In this case, the target signature is assumed to be deterministic and represented by its response under a flat interface. We obtain multiple data for hypothesis $H_{1}$ (target present) by superposing the flat-surface target signature with multiple clutter realization $\mathbf{c}$ obtained from multiple simulations of the rough interface. This idealized data is then processed with a matched filter, designed for the flat-surface target signature, yielding an upper bound on sensor performance (since in reality, as elucidated above, the target signature is random if the surface is rough). For this case, the ROC is based on the signal energy to clutter-variance ratio [11]. The second curve represents the actual matched filter performance, when the target signature is simulated rigorously under a rough interface, yielding $\mathbf{e}=\mathbf{c}+\mathbf{s}$ (with $\mathbf{s}$ random). In general, we see that the matched filter, designed for a flat-surface target response, performs quite poorly on the actual data. The third curve represents the performance of the optimal detector (Fig. 3), as applied to the rigorous data, with the perfor- 


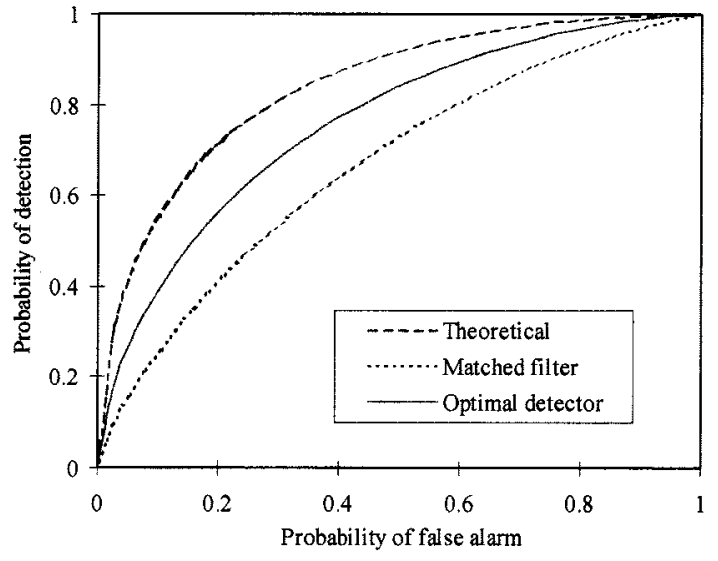

(a)

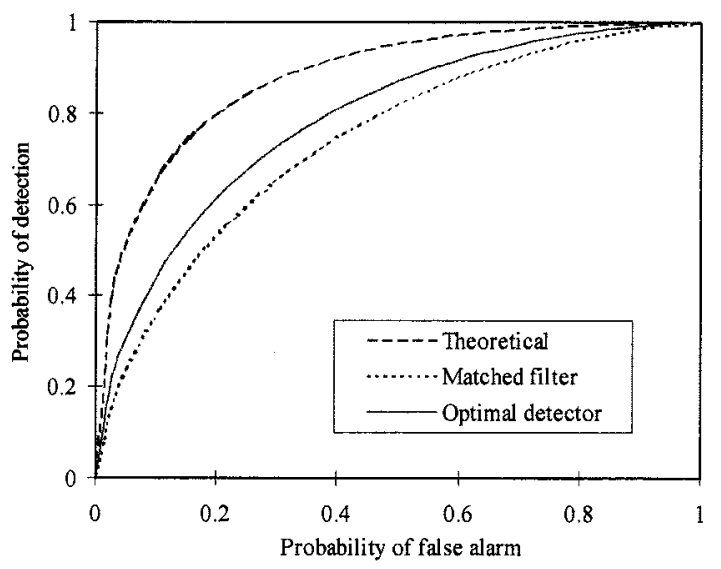

(b)

Fig. 18. Receiver operating characteristics for a fractal surface with $\sigma=6.25$ $\mathrm{cm}$ and $D=1.5$, incidence at $30^{\circ}$. (a) $T E$ polarization and (b) $T M$ polarization.

mance of this detector generally falling between the aforementioned two curves. In all cases, the data is prewhitened.

In the detector results, we consider the same physical parameters as addressed in Section V-B: Gaussian, exponential, and fractal surfaces, for both polarizations and incidence angles $30^{\circ}$ and $65^{\circ}$. In Figs. 12 and 13, we consider Gaussian surfaces. Figs. 14 and 15 address exponential surfaces, Figs. 16 and 17 consider Weierstrass fractal surfaces with $D=1.2$ and Figs. 18 and 19 investigate Weierstrass fractal surfaces with $D=1.5$. The statistical parameters of the above surfaces are as in Section V-B. As discussed above, the fractal surfaces constitute a different class of physics than the Gaussian and exponential surfaces. Therefore, in the discussions below, we compare the relative characteristics of the detector for the Gaussian and exponential surfaces, and separately examine detector performance for the fractal surfaces.

The difference in performance between the optimal detector and the matched filter is largely dictated by the amount of randomization induced in the fields as they are transmitted through the rough interface. If there is little randomization of the trans-

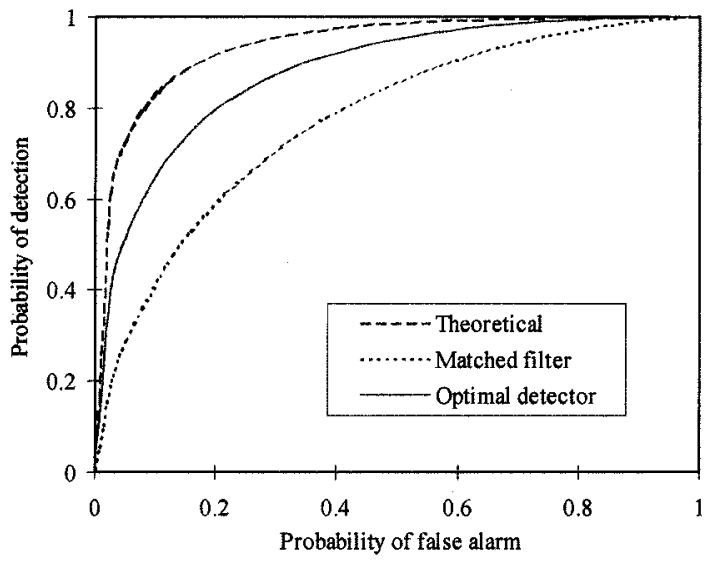

(a)

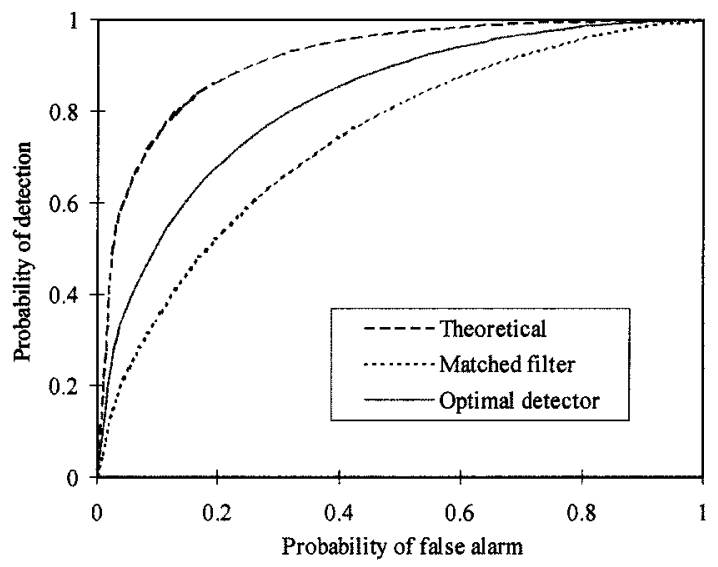

(b)

Fig. 19. Receiver operating characteristics for a fractal surface with $\sigma=6.25$ $\mathrm{cm}$ and $D=1.5$, incidence at $65^{\circ}$. (a) $T E$ polarization and (b) $T M$ polarization.

mitted incident fields, the target signature is nearly deterministic, for which the matched filter is optimal. To first order, the random surface manifests a local angle of incidence that varies randomly, as prescribed by the surface statistics. This is particularly true for relatively smooth rough surfaces, like the Gaussian surfaces. For such cases, the variation of the power transmitted into the ground, as a function of incidence angle, is therefore a good indication of this induced randomization. For the soil considered here, the variation of the transmitted power into the soil varies weakly for incident angles in the vicinity of $30^{\circ}$, for both polarizations. Consequently, for relatively smooth rough surfaces and an incident angle of $30^{\circ}$, there is minimal surface-induced randomization of the incident fields, and the matched filter (which assumes a deterministic targets signature) works relatively well. The TM-polarized plane wave has a Brewster angle of approximately $66^{\circ}$ for the soil considered, and therefore variation of the TM transmitted fields is weak for incident angles in the vicinity of $65^{\circ}$. By contrast, the TE-polarized fields have no Brewster angle, and there is substantial variation of the transmitted fields for angles in the vicinity of $65^{\circ}$. 
This is consistent with all the simulated results, which exhibit the largest optimal detector vs. matched filter performance improvement for $65^{\circ}$ and TE polarization [this is particularly evident in Fig. 13(a)].

We also note that, for the cases considered, detection performance is generally enhanced for incident angles closer to grazing (i.e., performance is generally better for $65^{\circ}$ incidence relative to $30^{\circ}$ ). As discussed previously, this is manifested because the ratio of target-signature energy to clutter energy is increased for larger incidence angles, for the examples examined. Concerning the relative detector performance for Gaussian and exponential surfaces, we note that the detector performance is similar for these two surfaces. However, we typically see more performance enhancement manifested by the optical detector for the Gaussian surface, relative to the exponential. This is because, for the same correlation length, the main difference between the Gaussian and exponential surfaces is more fine structure in the latter. The Gaussian surface is characterized by more large-scale variation, this having a greater impact on randomization of the transmitted fields over the bandwidth considered here. In particular, in Fig. 11 we notice that the Gaussian surface PSD has larger magnitude than the exponential surface in the region close to the central frequency of the incident pulse, where most of the electromagnetic energy is concentrated.

For the surfaces considered, the clutter from the Weierstrass fractal surfaces was considerably more intricate than that of the Gaussian and exponential surfaces considered (see Figs. 6-9). One would therefore expect that the fractal surfaces considered likewise randomize the transmitted fields more significantly than the Gaussian and exponential surfaces. This is manifested in Figs. 16-19, in which a noticeable increase in performance is realized via the optimal detector, vis-à-vis the matched filter. We note that the degree of randomization appears to be an intricate function of the incidence angle and the fractal dimension. In particular, we notice that the largest improvement in detector performance occurs at $65^{\circ}$ (for both polarizations), and that this improvement is more marked for the surfaces with $D=1.2$ than for $D=1.5$.

\section{CONCLUSIONS}

We have applied time-domain electromagnetic simulations to the problem of ultrawideband sensing of targets buried under a rough air-ground interface. Our main focus has been to examine time-domain electromagnetic scattering from three classes of surface statistics, and to examine the effects of such randomization on optimal subsurface target detection. In many cases, for which the target signature was significantly randomized, the optimal detection performance improvement, relative to a match filter, was substantial. For the surfaces considered, greater target-signature randomization was realized for the fractal surface, vis-à-vis the Gaussian and exponential surfaces. However, the details of these surfaces are very different, and therefore it is difficult to make direct comparisons.

There are many fertile areas of future research. In particular, the Haar-wavelet expansion represents the simplest realization of the MRTD model. One direction for future research consists of improving the numerical modeling scheme by considering other wavelet basis, with better smoothness properties. Another interesting direction involves studying scattering from Weierstrass fractal surfaces in greater detail, both analytically and numerically.

\section{REFERENCES}

[1] M. A. Ressler and J. W. McCorkle, "Evolution of the Army Research Laboratory ultra-wideband test bed," in Ultra-Wideband Short-Pulse Electromagnetics 2, L. Carin and L. B. Felsen, Eds. New York: Plenum, 1995, pp. 109-123.

[2] T. Dogaru and L. Carin, "Time-domain sensing of targets buried under a rough air-ground interface," IEEE Trans. Antennas Propagat., vol. AP-46, pp. 360-372, Mar. 1998.

[3] T. Dogaru, L. Collins, and L. Carin, "Optimal time-domain detection of a deterministic target buried under a randomly rough interface," IEEE Trans. Antennas Propagat., vol. 49, pp. 313-326, Mar. 2001

[4] M. Saillard and D. Maystre, "Scattering from metallic and dielectric rough surfaces," J. Opt. Soc. Amer. A, vol. 7, pp. 982-990, June 1990.

[5] E. I. Thorsos, "The validity of the Kirchoff approximation for rough surface scattering using a Gaussian roughness spectrum," J. Acoust. Soc. Amer, vol. 83, pp. 78-92, 1988.

[6] E. I. Thorsos and D. R. Jackson, "The validity of the perturbation approximation for rough surface scattering using a Gaussian roughness spectrum," J. Acoust. Soc. Amer, vol. 86, pp. 261-277, 1989.

[7] F. D. Hastings, J. B. Schneider, and S. L. Broschat, "A Monte-Carlo FDTD technique for rough surface scattering," IEEE Trans. Antennas Propagat., vol. 43, pp. 1183-1191, Nov. 1995.

[8] A. Taflove, Computational Electrodynamics: The Finite-Difference Time-Domain Method. Norwood, MA: Artech, 1995.

[9] M. Krumpholz and L. Katehi, "MRTD: New time-domain schemes based on multiresolution analysis," IEEE Trans. Microwave Theory Tech., vol. 44, pp. 555-571, Apr. 1996.

[10] T. Dogaru and L. Carin, "Multiresolution time-domain analysis of scattering from a rough dielectric surface," Radio Sci., vol. 35, pp. 1279-1292, Nov.-Dec. 2000

[11] H. L. Van Trees, Detection, Estimation, and Modulation Theory. New York: Wiley, 1968

[12] L. Tsang, G. F. Zhang, and K. Pak, "Detection of a buried object under a single random rough surface with angular correlation function in EM scattering," Microw. Opt. Tech. Lett., vol. 12, p. 375, Aug. 20, 1996

[13] G. F. Zhang and L. Tsang, "Angular correlation function of wave scattering by a random rough surface and discrete scatterers and its application in the detection of a buried object," Waves Random Media, vol. 7, pp. 467-478, July 1997.

[14] G. F. Zhang, L. Tsang, and K. Pak, "Angular correlation function and scattering coefficient of electromagnetic waves scattered by a buried object under a two-dimensional rough surface," J. Opt. Soc. Amer. A., vol. 12, pp. 2995-3002, Dec. 1998.

[15] G. F. Zhang, L. Tsang, and Y. Kuga, "Studies of the correlation function of scattering by random rough surfaces with and without a buried object," IEEE Trans. Geosci. Remote Sensing, vol. 35, pp. 444-453, Mar. 1997.

[16] I. Daubechies, Ten Lectures on Wavelets. Philadelphia, PA: SIAM, 1992.

[17] T. Dogaru, "Modeling and signal processing for electromagnetic subsurface sensing,", Ph.D. dissertation, Duke University, Durham, NC, 1999.

[18] R. F. Harrington, Field Computation by Moment Methods. Malabar, FL: Krieger, 1968.

[19] B. B. Mandelbrot, The Fractal Geometry of Nature. New York: W. H. Freeman, 1983

[20] D. L. Jaggard and Y. Kim, "Diffraction by band-limited fractal screens," J. Opt. Soc. Amer. A, vol. 4, pp. 1055-1062, June 1987.

[21] S. Rouvier, P. Borderies, and I. Chenerie, "Ultra-wideband electromagnetic scattering of a fractal profile," Radio Sci., vol. 32, pp. 285-293, Mar-Apr. 1997.

[22] E. Bachelier, C. Ruiz, P. Borderies, I. Chenerie, and M. Davidson, "Weierstrass functions determination for soil modeling," in Proc. Int. Geoscience and Remote Sensing Symp.'98, Seattle, WA, 1998.

[23] M. V. Berry and Z. V. Lewis, "On the Weierstrass-Mandelbrot fractal function," Proc. R. Soc. London, pp. 459-484, 1980.

[24] P. Hubral and M. Tygel, "Analysis of the Rayleigh pulse," Geophysics, vol. 54, pp. 654-658, 1989. 


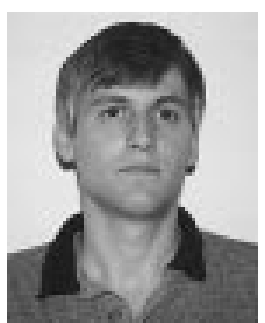

Traian Dogaru was born in Bucharest, Romania, in 1966. He received the engineering degree from the Polytechnic University of Bucharest in 1990 the M.S. degree in electrical engineering in 1997, and the Ph.D. degree in 1999, both from Duke University, Durham, NC

From 1992 to 1995, he held different engineering positions in the magnetic recording industry. He is currently a Research Associate with Duke University, and his main research interests are in electromagnetic wave theory, computational electromagnetics, rough surface scattering, and radar-related signal processing.
Lawrence Carin (SM'96-F'01) was born March 25, 1963, in Washington, DC, and received the B.S., M.S., and Ph.D. degrees in electrical engineering from the University of Maryland, College Park, in 1985, 1986, and 1989, respectively.

In 1989, he joined the Electrical Engineering Department, Polytechnic University, Brooklyn, NY, as an Assistant Professor, and became an Associate Professor there in 1994. In September 1995, he joined the Electrical Engineering Department at Duke University, Durham, NC, where he is now a Professor. He is the Principal Investigator on a Multidisciplinary University Research Initiative (MURI) on demining. His current research interests include short-pulse scattering, subsurface sensing, and wave-based signal processing.

Dr. Carin is a member of the Tau Beta Pi and Eta Kappa Nu honor societies and is currently an Associate Editor of the IEEE TRANSACTIONS ON ANTENNAS AND PROPAGATION. 